\title{
Efficient Detection of All Pointer and Array Access Errors
}

\author{
Todd M. Austin Scott E. Breach Gurindar S. Sohi \\ Computer Sciences Department \\ University of Wisconsin-Madison \\ 1210 W. Dayton Street \\ Madison, WI 53706 \\ \{austin, breach, sohi\}@cs.wisc.edu
}

\begin{abstract}
We present a pointer and array access checking technique that provides complete error coverage through a simple set of program transformations. Our technique, based on an extended safe pointer representation, has a number of novel aspects. Foremost, it is the first technique that detects all spatial and temporal access errors. Its use is not limited by the expressiveness of the language; that is, it can be applied successfully to compiled or interpreted languages with subscripted and mutable pointers, local references, and explicit and typeless dynamic storage management, e.g., C. Because it is a source level transformation, it is amenable to both compile- and run-time optimization. Finally, its performance, even without compile-time optimization, is quite good. We implemented a prototype translator for the $\mathrm{C}$ language and analyzed the checking overheads of six non-trivial, pointer intensive programs. Execution overheads range from $130 \%$ to $540 \%$; with text and data size overheads typically below $100 \%$.
\end{abstract}

\section{Introduction}

It is not difficult to convince programmers (or employers of programmers) that programming errors are costly, both in terms of time and money. Memory access errors are particularly troublesome. A memory access error is any dereference of a pointer or subscripted array reference which reads or writes storage outside of the referent. This access can either be outside of the address bounds of the referent, causing a spatial access error, or outside of the lifetime of the referent, causing a temporal access error. Indexing past the end of an array is a typical example of a spatial access error. A typical temporal access error is assigning to a heap allocation after it has been freed.

Our own experiences as programmers as well as published evidence lead us to believe that memory access errors are an important class of errors to reliably detect. For example, in

This work was supported by grants from the National Science Foundation (grant CCR-9303030) and Office of Naval Research (grant N00014-93-1-0465).
[MFS90], Miller et. al. injected random inputs (a.k.a "fuzz") into a number of Unix utilities. On systems from six different vendors, nearly all of the seemingly mature programs could be coaxed into dumping core. The most prevalent errors detected were memory access errors. In [SC91], Sullivan and Chillarege examined IBM MVS software error reports over a four year period. Nearly $50 \%$ of all reported software errors examined were due to pointer and array access errors. Furthermore, of these errors, $25 \%$ were temporal access errors an error our checking methodology is particularly adept at catching.

Memory access errors are possible in languages with arrays, pointers, local references, or explicit dynamic storage management. Such errors are particularly difficult to detect and fix because:

- The effects of a memory access error may not manifest themselves except under exceptional conditions.

- The exceptional conditions which lead to the program error may be very difficult to reproduce.

- Once the error is reproduced, it may be very difficult to correlate the program error to the memory access error.

Consider the erroneous $\mathrm{C}$ function in Figure 1. This function can create a memory access error in the return statement expression. The function will reference the word immediately following the array referenced by the pointer data if the array does not contain the token.

The function illustrates the three difficulties in finding and fixing memory access errors. First, FindToken() will only produce an incorrect result if the word following the array referenced by data contains the same value as token (or is inaccessible storage). This event is unlikely if the word contains an arbitrary value. Second, when (or if) FindToken() creates an incorrect result, it will be difficult to recreate during debugging. The programmer will have to condition the inputs of the program such that the word following the array referenced by data once again contains the same value as token. If the value of the illegally accessed word is independent of the value of token, the probability of success will be very low. Third, correlating the visible errors of the program to the incorrect actions of FindToken() may be very difficult. This connection may be very subtle and may not be visible for a long period of time.

Many execution environments do provide some level of protection against memory access errors. For example, in most Unix based systems, a store to the program text will cause the operating system to terminate execution of the program (usually with a core dump). Unix typically provides storage 


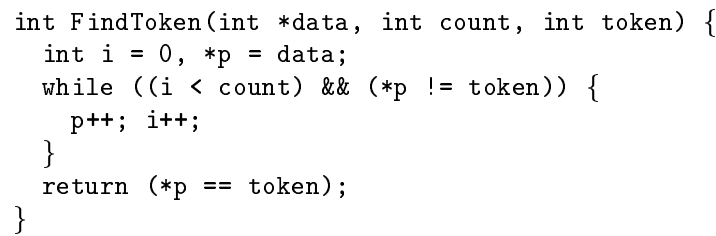

Figure 1: A C function with a (spatial) memory access error.

protection on a segment granularity - the segments are the program text, data, and stack. Other, more hostile environments such as MS-DOS, do not offer such luxuries, and stores to the program text may or may not manifest themselves as a program error. If a program error does occur, correlating it to a fault may be difficult, if not impossible.

Ideally, we would like the language execution environment to support memory access protection at the variable level, that is, an access to a variable should only be valid if the access is within the range (for both time and space) of the intended variable - all other accesses should immediately flag an error. We call any program that supports these execution semantics a safe program.

Our solution to the memory access error problem is simple and provides efficient and immediate detection of all memory access errors. We transform programs, at compile-time, to use an extended pointer representation which we call a safe pointer. A safe pointer contains the value of the pointer as well as object attributes. The object attributes describe the location, size and lifetime of the pointer referent. When a safe pointer value is created, either through the use of the reference operator (e.g., ' $\&$ ' in C) or through explicit storage allocation, we attach to it the appropriate object attributes. As the value is manipulated, through the use of pointer operators, the object attributes are transferred to any new safe pointer values. Detecting a memory access error involves simply validating dereferences against the object attributes - if the access is within the space and time bounds of the object, it is permitted, otherwise an error is flagged and the access error is detected immediately.

We implemented a prototype source-to-source translator for the $\mathrm{C}$ language and examined the performance of six nontrivial, pointer intensive programs. The performance is quite good. Instruction execution overheads range from $130 \%$ to $540 \%$, and text and data size overheads are typically below $100 \%$. We also benchmarked our prototype system against two commercially available tools that support memory access checking (Purify [HJ92] and CodeCenter [KLP88]) and found that our checking technique consistently uses less resources, even while providing better error coverage for memory access errors.

This paper is organized as follows. Section 2 introduces our extended safe pointer representation. Section 3 details the program transformations required to create safe programs, and in Section 4 we discuss the translation and performance implications of providing complete error coverage. In Section 5 , we present compile- and run-time optimization frameworks. Section 6 describes our prototype implementation and presents results of our analyses of six programs. Section 7 compares our checking technique to other published techniques. Section 8 concludes the paper.

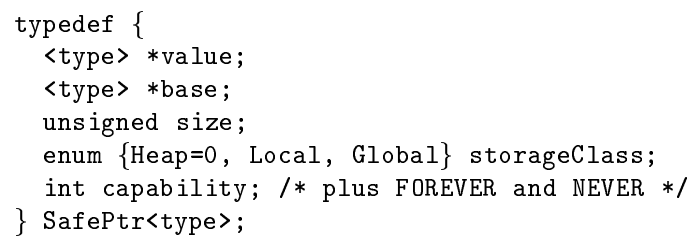

Figure 2: Safe pointer definition. This C-like type definition is parameterized by <type>, the type of the pointer referent.

\section{Safe Pointers}

To enforce access protection, we must extend the notion of a pointer value to include information about the referent. The idea is similar to tagged pointers used in many Lisp implementations [Lee91]. Figure 2 shows our safe pointer representation. The definitions of the contained fields follow:

value: The value of the safe pointer; it may contain any expressible address.

base and size: The base address of the referent and its size in bytes. In languages where pointers are immutable, base is redundant and may be omitted. With this information, we can detect all spatial access errors with a range check.

storageClass: The storage class of the allocation, either Heap, Local, or Global. Using this value, it is possible to detect errant storage deallocations, e.g., it is illegal to free a global or local variable.

capability: A capability to the referent. When dynamic variables are created, either through explicit storage allocation (e.g., calls to malloc()) or through procedure invocations (i.e., a procedure call creates the local variables in the stack frame of the procedure), a unique capability is issued to that storage allocation. The unique capability is also inserted into an associative store called the capability store and deleted from that store when the dynamic storage allocation is freed or when the procedure invocation returns (the exact mechanics of this process are discussed in a following section). Thus, the collection of capabilities in the capability store represent all active dynamic storage. Temporal access errors occur whenever a reference is made through a stale pointer, i.e., a pointer which references storage whose capability is no longer in the capability store. Two capabilities are predefined. FOREVER is unique and always exists in the capability store; this capability is assigned to all global objects. NEVER is unique and never exists in the capability store; this capability can be assigned to invalid pointers to ensure any dereference causes an error.

The value attribute is the only safe pointer member that can be manipulated by the program source; all other members are inaccessible. base and size are the spatial attributes. storageClass and capability are the temporal attributes.

Safe pointers can exist in three states: unsafe, invalid, and valid. If the object attributes are incorrect, we say that the pointer has become unsafe; dereferencing this pointer may cause an undetected memory access error. It is the goal of this work to ensure that a safe pointer never becomes 
unsafe. If the safe pointer is not unsafe, it is either invalid or valid, depending on whether a dereference would flag an error. Languages with mutable pointers allow the program to legally create invalid pointers; for example, iterating a pointer across all the elements of an array exits the loop with the pointer pointing to the memory location following the last object. If the invalid pointer is never dereferenced, the program would not be in error. This behavior illustrates precisely why we only place error checks at dereferences; it is not illegal to have an invalid pointer - only to use it.

The initial value of a safe pointer, if not specified by an initialization expression, must be invalid. This condition ensures that a dereference before the initial assignment is detected. A simple way to invalidate a pointer value is to assign it the unique capability NEVER.

\section{Program Transformations}

Creating a safe program from its unsafe counterpart involves three transformations: pointer conversion, check insertion, and operator conversion. The first, pointer conversion, extends all pointer definitions and declarations to include space for object attributes. Check insertion instruments the program to detect all memory access errors. Operator conversion generates and maintains object attributes. In this section, we also describe the run-time support.

\subsection{Pointer Conversion}

All pointer definitions and declarations must be extended to include object attributes. To make this transformation transparent, the composite safe pointer must mimic the first class value semantics of scalar pointers. That is, when passed to a function, the safe pointer must be passed by value, and when operators are applied to a safe pointer, the result, if a pointer, must be a new safe pointer.

There is no need to add object attributes to array variables. Array variables (in the $\mathrm{C}$ sense) are merely address constants, and thus only exist as statically allocated objects or within structure definitions; as a result, the spatial attributes can be generated from the address constant and its type size, and the temporal attributes can be taken from the safe pointer to the containing object or derived from the array name.

\subsection{Check Insertion}

Assuming the safe pointer object attributes are correct (how to ensure this property is detailed in the following sections), complete safety for all pointer and array accesses is provided by inserting an access check before each pointer or array dereference.

The dereference check first verifies that the referent is alive by performing an associative search for the referent's capability. If the referent has been freed, the capability would no longer exist in the capability store and the check would fail. Because capabilities are never re-used, the temporal check fails even if the storage has been reallocated. Once the storage is known to be alive, a bounds check is applied to verify that the entire extent of the access fits into the referent.

Our access check, shown in Figure 3, takes advantage of the wrap-around property of unsigned arithmetic to simplify

\footnotetext{
${ }^{1}$ We use the term dereference as a blanket term for any indirect access - either through application of the dereference operator (e.g., ' $*$ ' or ' $\rightarrow$ ' in C) or through indexing an array or pointer variable (e.g., '[ ]' in $\mathrm{C}$ ).
}

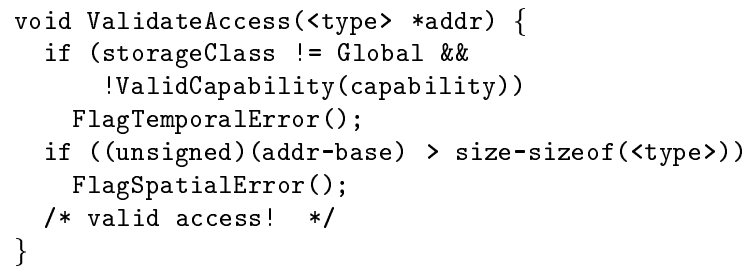

Figure 3: Memory access check. ValidCapability() indicates whether or not the passed capability is currently active, i.e., in the capability store. The Flag functions performs system specific handling of an access error.

the bounds check. If the accessed address is prior to the start of the array, the unsigned subtraction underflows and creates a very large number, causing the test to fail. The advantage of this expression over traditional bounds checks ${ }^{2}$ is that it only requires one conditional branch to implement. This simplification reduces the additional control complexity introduced by dereference checks, which can result in better optimization results and better dynamic executions.

\subsection{Operator Conversion}

Pointer operators must interact properly with the composite safe pointer structure. When applied, they must reach into the safe pointer to access the pointer value. If the operator creates a new pointer value, it must include an unmodified copy of the pointer operand's object attributes. For example, in the $\mathrm{C}$ statement $\mathrm{q}=\mathrm{p}+6$, the application of the ' + ' operator on the pointer $p$ creates a new safe pointer which is assigned to $\mathrm{q}$. The new pointer value in $\mathrm{q}$ shares the same object attributes as p. Operators which manipulate pointer values never modify the copied object attributes because changing the value of the pointer does not change the attributes of the storage it references. This property holds even for pointers to aggregate structures. In this case, the object attributes refer to the entire aggregate.

The assignment operator requires special handling if the right hand side is a constant. Two common pointer constants are the NULL value and pointers to string constants (for C). If the assignment value is NULL, the NULL value can be replaced by an invalid safe pointer value, e.g., one with the capability NEVER. For string constants, we can generate the needed object attributes at compile-time. If the right hand side of the assignment is a pointer expression, the resulting pointer value (and its object attributes) is copied to the pointer named on the left hand side of the assignment.

Casting between pointer types does not require any special program transformations. This operation only alerts the compiler that future pointer arithmetic or dereferences of a particular pointer value should be made with respect to the new type size. Casting to a non-pointer type requires that the object attributes be dropped (if only pointers carry object attributes) and the cast be carried out as defined by the language. Casting from a non-pointer type to a pointer type is problematic if non-pointer types do not carry object attributes. We address this problem in Section 4.

${ }^{2}$ Our check is functionally equivalent to:

(addr < base || addr > base+size-sizeof (<type〉))

which requires two conditional branches (or extra instructions to combine the boolean terms). 
Handling of the reference operator, e.g., the ' $\&$ ' operator in the C statement $q=\& p->b[10]$, is slightly more complex as it must generate object attributes. The reference operator is applied to an expression ( $\mathrm{p}->\mathrm{b}[10]$, in our example) which names some storage. We call this expression the $a c$ cess path. The result of the operation is a new safe pointer to the referent named by the expression.

To generate object attributes for a reference operation (e.g., '\&'), we decompose access paths into two parts, a prefix and a suffix. The access path prefix is a non-empty sequence of variable names, dereferences, subscripts, field selectors, and pointer expressions leading to the memory object being referenced. The remaining part of the access path, the access path suffix, is a possibly-empty sequence of field selectors and subscripts (on array variables only) indicating the extent of the memory object being referenced.

We further classify access paths as direct or indirect. A direct access path refers to an object in the global or local space by name. An indirect access path contains at least one pointer traversal.

Given a reference operator expression, we can parse the access path prefix by traversing the expression tree starting with the left-most, lowest precedence operator. The part of the expression up to but not including the last pointer traversal is the access path prefix; the remainder of the expression becomes the access path suffix. If the access path does not contain any pointer traversals, the access path prefix is merely the name of the referenced variable.

To illustrate this decomposition, consider the $\mathrm{C}$ expression $\& f->g[4] . i[6]$, where $g$ is a pointer and $i$ is an array within a structure. The access path prefix is the sub-expression $f->g[4]$. The access path suffix is the remainder of the expression, $i[6]$. The access path prefix is indirect.

The temporal attributes of the new safe pointer are derived from the access path prefix. If the prefix is direct, the referenced object is either a global or a local variable. If global, the capability FOREVER is assigned to the new safe pointer. If local, the capability allocated to the local variable's stack frame is assigned to the new safe pointer (frame capability allocation is discussed in the following section). If the access path prefix is indirect, the temporal attributes are taken from the safe pointer named by the access path prefix.

The spatial attributes are derived from both the access path prefix and suffix. The base of the safe pointer is taken from the object referred to by the access path prefix, namely the address of the named variable for a direct prefix or the corresponding spatial attributes of the referenced safe pointer for an indirect prefix. The value and size of the safe pointer are computed from the access path suffix. Because all members of the referenced object (i.e., the member of any contained structure) are of a known size, the spatial attributes of the reference can be computed at compile-time from type information. In the event the final term of the suffix is a subscript, the spatial attributes are set to the extent of the entire array. This technique allows the safe pointer to be subsequently manipulated to point to other members of the array.

The use of the access path prefix and suffix to produce a safe pointer via the reference operator cannot subvert the checking framework. In order to maintain safe semantics, any pointers traversed within the access path prefix must be validated using the techniques described in the previous subsection.

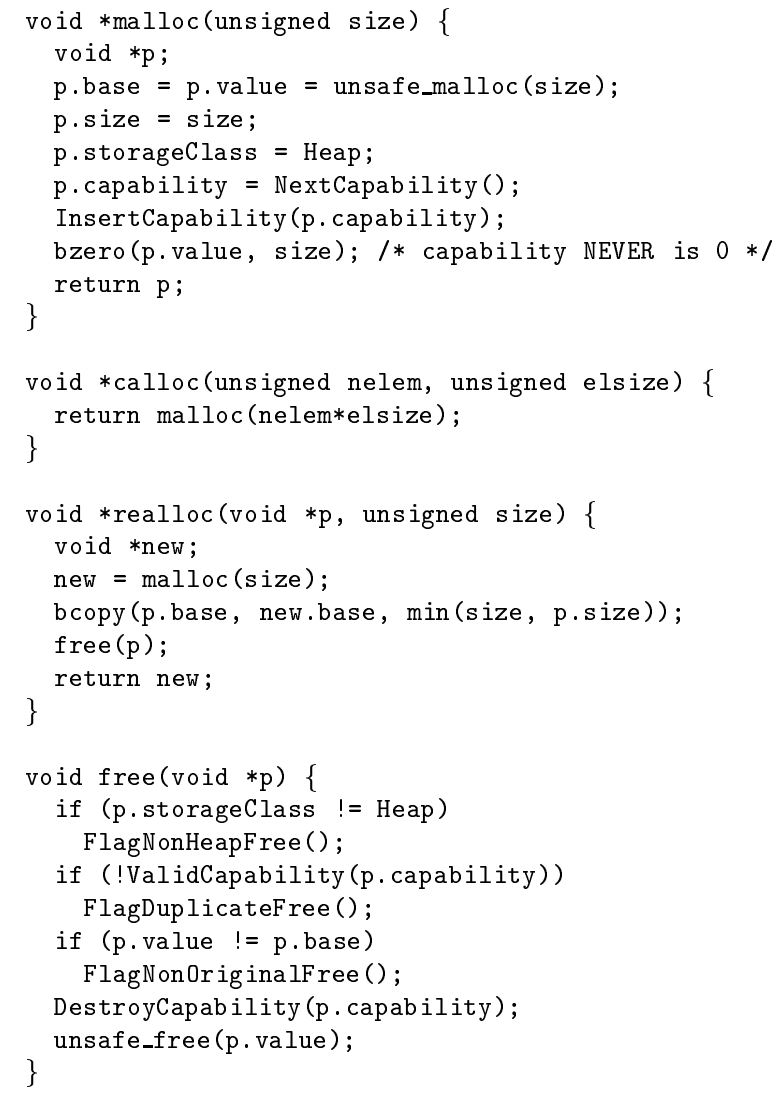

Figure 4: Safe malloc implementation with additional checking. InsertCapability(), ValidCapability(), and DestroyCapability() insert, locate, and delete capabilities, respectively. NextCapability() returns the next unique capability. unsafe_malloc() and unsafe_free() are interfaces to the systemdefined storage allocator.

\subsection{Run-Time Support}

The explicit storage allocation mechanism must be extended to create safe pointers. During allocation, a capability must be allocated for the storage, and any contained pointers must be invalidated. At deallocation, the capability given to the storage must be destroyed.

Figure 4 shows how this support would be provided for malloc(), the storage allocator provided under Unix. During allocation, malloc() generates a safe pointer using the size and location of the allocation request. The call to NextCapability() returns the next available and unused capability. NextCapability() can be implemented with an incrementing counter or a pseudo-random number generator. The capability is inserted into the capability store via the call to InsertCapability(). The call to bzero() clears the entire storage allocation. This action ensures that any pointers in the untyped allocation are initially invalid (assuming the storage class of Heap and capability NEVER are both assigned the value of 0 ).

The implementation of realloc() is slightly more subtle. This function takes an existing storage allocation and resizes it to the requested size. The reallocated storage may move for any request, either larger or smaller. If moved, the contents of the new allocation will be unchanged up to the lesser of the new and old sizes. In our safe programming environment, we 


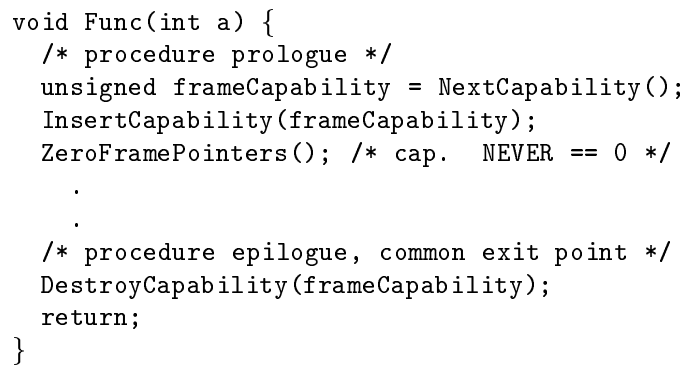

Figure 5: Function frame allocation and deallocation. ZeroFramePointers () is a system specific function which clears all pointers in the newly allocated stack frame.

must move the storage in all cases, otherwise, there may exist safe pointers (which we cannot locate and change) whose object attributes have incorrect records of the referent size. If dereferenced, these pointers may flag errors even though the access was valid in the reallocated storage, or worse, the reallocation may have shrunk the referent, creating unsafe pointers whose referent sizes are too large. We can solve both these problems by always moving the storage. This action will force the program to update any old pointers to the previous allocation. Because the reallocated storage is allocated under a new capability, any stale pointers to the previous allocation will flag errors if dereferenced. We need not clear the remaining storage in the reallocation if it is larger, as the call to malloc() returns cleared storage.

At calls to free(), the capability of the allocation (contained in the safe pointer object attributes) is deleted from the capability store by the call to DestroyCapability(). Our implementation also verifies that the freed storage is indeed a heap allocation, has not been previously freed, and points to the head of the allocation (as this condition is required by free()).

The same allocation mechanism is applied to the dynamic storage allocated in procedure stack frames. When a function is invoked, a capability must be allocated for the entire frame if it contains any referenced locals. Any pointers contained in the frame must be set to an invalid state.

Figure 5 shows how this rewriting would be done for a $\mathrm{C}$ function. The function ZeroFramePointers () serves the same purpose as the call to bzero() in malloc(); it ensures that any pointers in the procedure stack frame are initially invalid by clearing the frame storage. Because stack frame allocations are strongly typed, ZeroFramePointers() could be replaced by NULL assignments to all the frame pointers.

If the language supports non-local jumps, e.g., longjmp() in $\mathrm{C}$, the run-time support must delete the frame capabilities of any elided function frames. This operation can be simply and portably implemented if the local capability space and heap capability space are kept disjoint, and function frame capabilities are allocated using an incrementing counter. The allocation of frame capabilities then becomes a depth-first numbering [ASU86] of the dynamic call graph. When a nonlocal jump occurs, all elided frame capabilities between the source frame and destination frame are deleted by removing all frame capabilities in the capability store that are larger than the frame capability of the destination frame. This mechanism only works if the source and destination frames are on the same call stack - this stipulation may not be true in all cases, e.g., coroutine jumps.

The capability store is an associative memory containing the capabilities of all active memory. It can be implemented as a hash table with the capability as the hash key. Accesses to the capability store exhibit a great deal of temporal locality, so moving accessed elements to the head of the hash table bucket chains is likely to decrease average access time.

We close this section with two examples. Figure 6(a) shows a spatial access error, and Figure 6(b) demonstrates a temporal access error. Safe pointer values are specified as a 5-tuple with the following format: [value,base,size, storageClass, capability]. $x$ indicates a don't care value. In the first example, a spatial access error is flagged when the program dereferences a safe pointer whose value is less than the base of the referent. In the second example, a stale pointer, q, is dereferenced. Even though the same storage has been reallocated to $\mathrm{p}$, the capability originally assigned to $\mathrm{q}$ has been destroyed during the call to free (); thus, the temporal access error is detected.

\section{Implications of Complete Error Coverage}

Our safe programming technique can detect all memory access errors provided that the following conditions hold:

$i$. Storage management must be apparent to the translator.

ii. The referents of all pointer constants must have a known location, size, and lifetime.

iii. The program must not manipulate the object attributes of any pointer value.

Our claim to complete error coverage must be limited to storage management controlled by the safe programming run-time system. If a program implements a domain specific allocator at the user level, some memory access errors, as viewed by the programmer, can be missed.

Consider, for example, a fixed size storage allocator. If a program relies heavily on a fixed size structure, storage requirements and allocation overheads can be greatly reduced by applying a fixed size allocation strategy. At the program level, the fixed size allocator calls the system allocator, e.g., malloc() or sbrk(), to allocate a large memory allocation. The fixed size allocator then slices the system allocation into fixed size pieces with a zero overhead for each allocation. Under this scheme, our safe programming technique would ensure that no accesses to a fixed size allocations are outside of the space and time bounds of the block from which the fixed size allocation was derived. This imprecision occurs because the translator can not distinguish the user level storage allocation actions from other pointer related program activities.

With some programmer intervention this problem can be overcome. Any useful safe compiler implementation will have to include an application programmers interface, or API, through which systems programmers can construct and manipulate the object attributes of safe pointers. In the case of the fixed size storage allocator, the programmer would specify the base and size of the fixed size allocation. The storage class and capability would be generated from the safe pointer to the block from which the fixed size allocation was derived.

Without the second qualification, the compiler may not be able to generate correct object attributes for a pointer constant. For example, device driver code typically creates pointers to device buffers and registers by recasting an integer to a pointer value. The translator has no way of knowing 

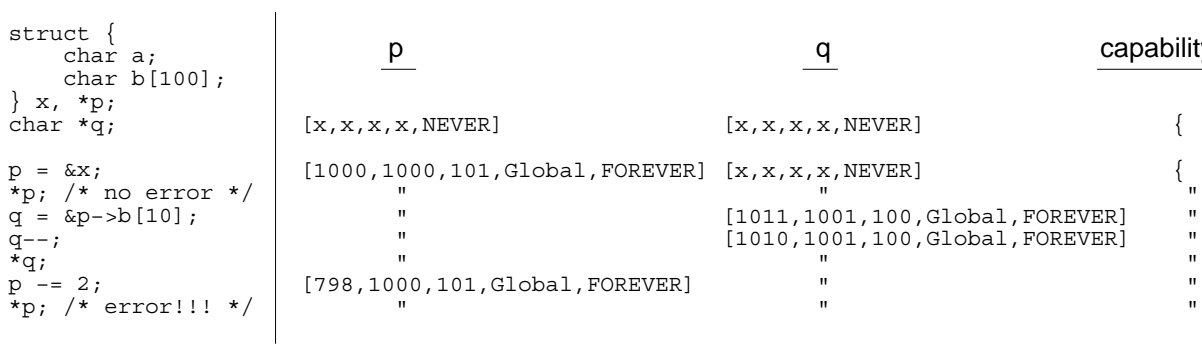

a)

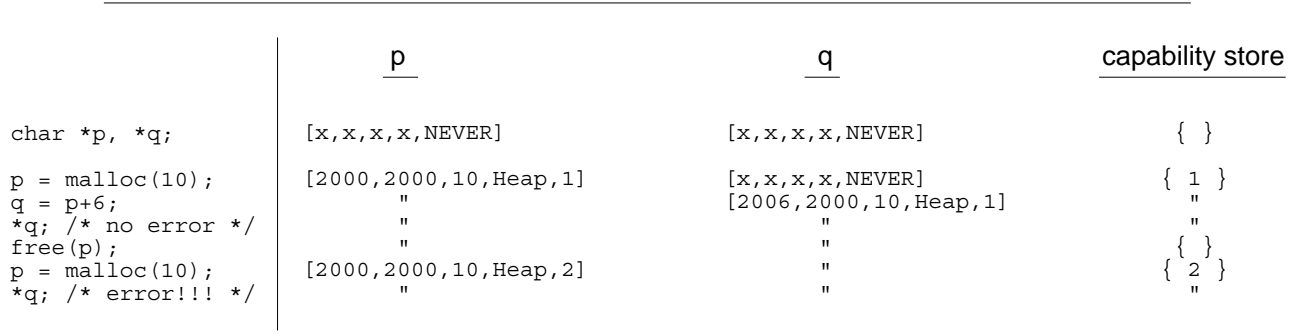

b)

Figure 6: Memory access checking examples. Figure a) is an example of a spatial access error, Figure $b$ ) is an example of a temporal access error. Safe pointer values, shown after each line is executed, are specified as a 5-tuple with the following format: [value,base,size, storageClass,capability]. An occurrence of $x$ indicates a don't care value.

the size and lifetime of the referent; thus, program safety cannot be maintained. In $\mathrm{C}$, the only well defined pointer constants are NULL, strings, and functions. For all other cases, this problem can be avoided by supplying the programmer with an API suitable for specifying the size and lifetime of problematic pointer constants.

The second qualification does not, however, preclude the use of recasts from non-pointer variables to pointer variables. To successfully support these operations, object attributes must be attached to all variables. In general, to provide complete safety, we need to attach object attributes to any storage that could hold a pointer value. It is our contention that most "well behaved" programs will only require pointer variables to carry object attributes.

The final qualification protects object attributes. If a program can arbitrarily manipulate the object attributes of a pointer value, then safety can always be subverted. For example, changing the storage class of a pointer from Global to Heap and then freeing the pointer would likely cause disastrous effects under our storage allocation scheme.

If object attributes are only attached to pointer values, the danger exists of manipulation through the use of recasts or unions. With a recast, it is possible to type storage in the referent first as a non-pointer value, manipulate the storage arbitrarily, and then recast the referent storage to a (possibly unsafe) pointer. Using a union, it is possible to create a pointer value under one field and then manipulate the object attributes of the pointer value through another overlaid, nonpointer field of the union.

The only solution that we can conceive to prevent this kind of manipulation is to attach object attributes to each byte of allocated storage. For types larger than one byte, the object attributes would be copied to all other storage holding the allocation. In this way, any arbitrary overlaying of types would still not allow the object attributes to be manipulated at the program level.

In reality, we can provide a high margin of safety for "well behaved" programs by attaching object attributes only to pointer values. We consider a well behaved program to be one in which pointer values are never created from or manipulated as non-pointer values. If a program violates this rule intentionally (e.g., through a recast), a safe compiler which makes a conservative approximation as to the intended referent of the new pointer value allows the pointer to access any live storage. ${ }^{3}$ If the rule is broken unintentionally (e.g., through incorrect use of a union), the error will likely be caught because it is difficult to manufacture, accidentally, an unsafe pointer.

\section{Optimizing Dereference Checks}

In the interest of performance, it may be possible to elide dereference checks and still provide complete program safety. If we can determine that the following invariant holds, the check may be elided.

A check at a dereference of pointer value $v$ may be elided at program point $p$ if the previous, equivalent check executed on $v$ has not been invalidated by some program action.

We can implement this check optimization either at runtime or at compile-time. Run-time check optimization has the advantage of being more flexible. We only need to execute the checks absolutely required to maintain program safety. However, the cost for this precision is extra safe pointer state which must copied, maintained, and checked at each dereference. Compile-time check optimization, on the other hand, is less flexible because we must constrain the decision to elide a check to all previous possible executions leading to a program point. The advantage of compile-time

\footnotetext{
${ }^{3}$ Note that in this case, safety can no longer be guaranteed because the intended referent is not known. Hence, we cannot bind the object attributes of a live variable to the new pointer because the program may have manipulated the pointer value to point outside of the intended referent prior to recasting it to a non-pointer value.
} 


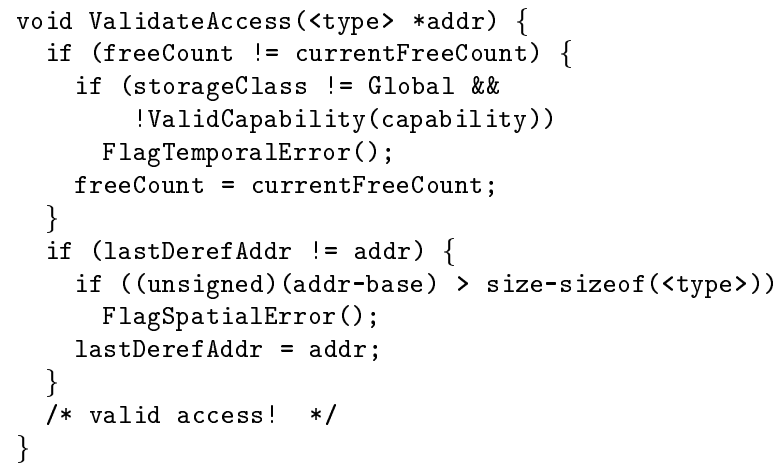

Figure 7: Memory access check with run-time check optimization. The variable currentFreeCount is a global counter incremented each time storage is deallocated.

check optimization is that no additional overhead is required at run-time to determine if a check may be elided.

\subsection{Run-Time Check Optimization}

We have designed and implemented a framework for dynamically eliding spatial and temporal checks. Spatial checks have no side effects, thus we can employ memoization [FH88] (or function caching) to elide their evaluation. We store the operands to the last check in the safe pointer object attributes, which amounts to the effective address of the last dereference. At any dereference, the spatial check may be elided if the effective address since the last check has not changed. This test is shown in Figure 7 in the if statement surrounding the bounds check. It may be useful to memoize more than one set of operands. In our implementation, we memoize both the effective address of the last dereference, i.e., use of the $\mathrm{C}$ operator '*', and the effective address of the last subscript operation, i.e., use of '[ ]'. Changes in the former can be tracked with only a single "dirty" bit, set when the pointer value is changed. Changes in the latter are tracked by retaining a copy of the last index applied to the pointer value.

To elide temporal checks, we keep a copy of a global counter, incremented when storage is deallocated, in the safe pointer. If this counter, which we call the free counter, has not changed since the last temporal check, the referent has not been freed and the temporal check can be safely elided. In our implementation, we keep separate counters for heap and stack deallocations.

\subsection{Compile-Time Check Optimization}

We have also designed (and are currently implementing) a compile-time optimization framework like that proposed by Gupta [Gup90]. Our algorithm implements a forward dataflow framework similar to that used by common subexpression elimination [ASU86]. However, our algorithm extends previous work to include eliding of temporal error checks, and because of our simplified bounds check, there is no need to split the optimization into upper and lower bounds check elimination.

Our optimization algorithm is shown in Figure 8. The algorithm is run twice, once for optimization of spatial checks and again for temporal checks. The algorithm executes in three phases.
Input: A flow graph $G$ with blocks $B$ with gen $\left[B_{i}\right]$ and $\operatorname{kill}\left[B_{i}\right]$ computed for each block $B_{i} \in B . \operatorname{gen}\left[B_{i}\right]$ is the set of check expressions generated in $B_{i}$. kill $\left[B_{i}\right]$ is the set of check expressions killed in $B_{i}$. The entry block is $B_{1}$. Output: A flow graph $G$ with redundant checks deleted. Method: The following procedure is executed twice, once for spatial check optimization and again for temporal check optimization.

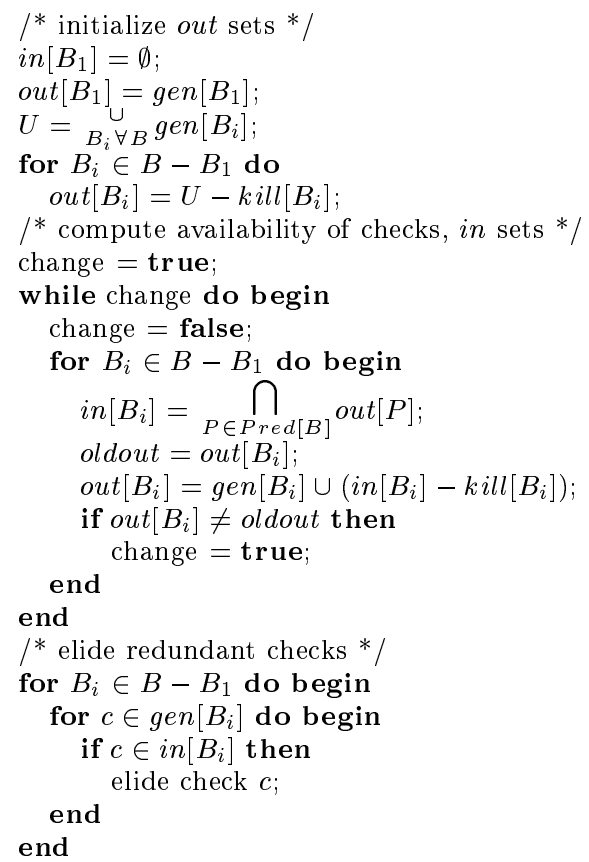

Figure 8: Compile-time check optimization algorithm.

In the first phase, the algorithm seeds the data-flow analysis by approximating all out sets. For all blocks except the entry block, the value of out $\left[B_{i}\right]$ is set to all check expressions less those killed by the block $B_{i}$, i.e., $U-k i l l\left[B_{i}\right]$. For the program entry block, $B_{1}$, we must assume that no checks are available, hence, $\mathrm{in}\left[B_{1}\right]$ is set to empty and out $\left[B_{1}\right]$ is set to the checks generated in the entry block $B_{1}$.

In the second phase, the data-flow framework is solved to determine where check expressions reach in the program. For a check expression to reach a node $B_{i}$, it must be available at $B_{i}$ for all executions, that is, it must be available in the out sets of all predecessors to block $B_{i}$. This requirement is precisely why the confluence operator is intersection. After the data-flow computation converges on a solution, i.e., change $==$ false, the set $i n\left[B_{i}\right]$ contains all checks that reach block $B_{i}$.

In the third phase, the in sets are used to elide redundant checks. Checks may be elided wherever a lexically identical or equivalent (if more powerful tests are applied) check is available in the block (i.e., the same check is in the $i n$ set of the block).

The defining feature for each analysis (spatial and temporal) is the specification of what constitutes a kill. A spatial check is killed by any assignment to a check operand, which includes assignment to the pointer variable or any of the operands of the index expression (if the pointer was indexed in the check expression). A temporal check is killed by any deallocation of the referent storage. If the referent of a free can be determined to be different than the check 
referent (e.g., through alias analysis), the free need not kill the check.

While performing these analyses, we must also be wary of kills that may occur through function calls or aliases. In either case, we must make a conservative approximation if insufficient information is available and assume that a kill does occur.

\section{Experimental Evaluation}

We evaluated our safe programming methodology by implementing a semi-automatic source-to-source translator and examining the run-time, code and data size overheads for six non-trivial programs. For each program, we analyzed its performance without optimization and with run-time resolved optimizations; we did not consider compile-time optimizations (our current implementation does not support this technique, though work in this direction is in progress).

\subsection{Experimental Framework}

We translated $\mathrm{C}$ programs to their safe counterparts by first rewriting all pointer and array declarations, calls to malloc() and free(), and references (use of the ' $\&$ ' operator) to use our Safe- $C$ macros. These macros, when passed through the $\mathrm{C}$ preprocessor $(C P P)$, produce either the original $\mathrm{C}$ program or a Safe-C program. A Safe-C program has all pointer and array declarations changed to type parameterized $\mathrm{C}++$ class declarations. Using operator overloading in the $\mathrm{C}++$ class definition, we implement the extended safe pointer and array semantics as described in Section 3.

All explicit storage allocation, i.e., calls to malloc() and free(), call wrapper functions which create safe pointers from the standard library routines. Our malloc() implementation clears all allocated storage, so any contained pointers start in the invalid state. If a local in a function is used as a pointer referent, we also rewrite the function to allocate a capability for the frame. Any pointer in the stack frame of a function is initialized to an invalid state in the constructor of the $\mathrm{C}++$ safe pointer class. Application of the reference operator calls a function which creates a safe pointer from the decomposed access path.

\subsection{Analyzed Programs}

We analyzed six programs, selected because each exhibits a high frequency of indirect references. The programs include an anagram generator (Anagram), a neural net simulator (Backprop), an arbitrary precision calculator (GNU $\mathrm{BC}$ ), a minimum spanning tree generator (Min-Span), a graph partitioning tool (Partition), and a VLSI channel router (YACR-2). Table 1 details the programs that we analyzed. For each, we show the code size (Instructions/Static), the number of instruction executed without checking (Instructions/Dynamic), the frequency of dereferences in the program text (Insts per Dereference/Static), and the dynamic frequency of dereferences executed (Insts per Dereference/Dynamic).

All programs were compiled and executed on a DECstation 3100 using AT\&T USL cfront version 3.0.1. The output of $c$ front (C code) was compiled using MIPS $c c$ version 2.1 at optimization level '-O2'. All instruction counts were obtained with QPT [Lar93].

For all analyses, object attributes were only attached to pointer values. We used a 15 byte safe pointer ( $275 \%$ overhead) in the unoptimized case: 4 byte pointer value, 4 byte

\begin{tabular}{||l|r|r|r|r||}
\hline \multirow{2}{*}{ Program } & \multicolumn{2}{|c|}{ Instructions } & \multicolumn{2}{c||}{ Insts per Dereference } \\
\cline { 2 - 5 } & $\begin{array}{c}\text { Static } \\
\left(\mathrm{x} 10^{3}\right)\end{array}$ & $\begin{array}{c}\text { Dynamic } \\
\left(\mathrm{x} 10^{6}\right)\end{array}$ & Static & Dynamic \\
\hline \hline Anagram & 10.0 & 19.4 & 106.3 & 7.6 \\
\hline Backprop & 10.8 & 122.4 & 148.5 & 8.9 \\
\hline GNU BC & 19.5 & 12.2 & 15.5 & 7.6 \\
\hline Min-Span & 11.9 & 13.3 & 48.7 & 5.9 \\
\hline Partition & 13.5 & 21.1 & 62.4 & 3.7 \\
\hline YACR-2 & 18.5 & 546.2 & 37.1 & 14.0 \\
\hline
\end{tabular}

Table 1: Analyzed programs.

base, 4 byte size, a 1 byte storage class specifier, and a 2 byte capability. For run-time check optimization, we added a 1 byte dirty flag, a 4 byte last index, and a 2 byte free counter for a total size of 22 bytes ( $450 \%$ overhead). Due to a bug in the $\mathrm{C}++$ compiler, we could not use sizeof () in the safe pointer implementation if the referent referred to itself; as a result, BC, Min-Span, and Partition all required the size of the referent to be stored in the safe pointer, which added a 4 byte overhead for these programs. There were no space overheads for array variables, as all required object attributes are known at compile-time. We only rewrote the actual program code, all system library routines remained unchecked. We did, however, perform interface checking. Whenever a system library is called, any pointer arguments are validated against the time and space bounds expected by the library routine. For example, if a call were made to fread(), the interface check would ensure that the destination of the read was live storage and that the entire length of the read operation would fit into the referent.

\subsection{Results}

Figure 9 shows the execution overheads for the analyzed programs. The Unopt columns show total dynamic instruction counts for executions with no optimization, and the $O p t$ columns show instruction counts with run-time resolved optimization.

For the run-time optimized executions, the normalized instruction counts range from $2.3(Y A C R-2)$ to $6.4(B C)$. This overhead reflects program performance without any compiletime optimization. While this performance degradation will likely be acceptable for the development cycle of short or medium length program executions, it may still be prohibitively expensive for very long running programs, and it is certainly too costly a price to pay for in-field instrumentation of a program. Examining more closely the breakdown of the execution overheads yields much insight into how the performance of our checking methodology could be improved.

For each program, we break down the overhead costs into five categories. We measured this cost by compiling and running the program repeatedly with incrementally more functionality in the safe pointer implementation. Original Program is the instruction count for the unchecked program, always normalized to one.

User Defined Ptr is the cost in our framework for implementing all pointers as structures at the user level. The primary factors affecting performance here are increased loads, stores, and function calls. The first factor is due to the MIPS $c c$ compiler's handling of structure variables; once wrapped in a structure, the field variables are no longer eligible for reg- 


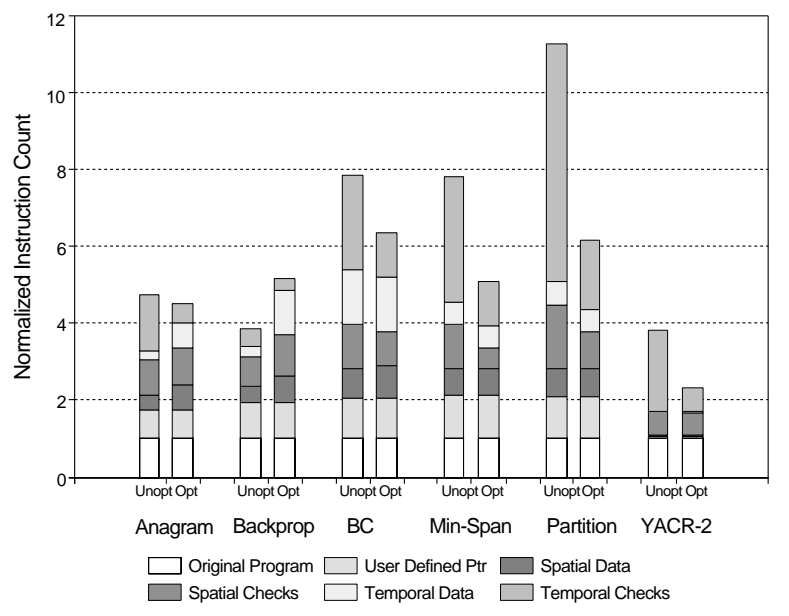

Figure 9: Execution overheads.

ister allocation. Wrapping attributes around pointers also increases the costs of procedure call parameter passing. MIPS $c c$ compiler passes most scalar arguments through registers; however, composite structures are always passed through memory (on the stack). The second major factor affecting performance is an increased number of function calls. The AT\&T $\mathrm{C}++$ compiler simplifies complex expressions created during template instantiation by extracting portions of the expression into static functions. This cost is only a side-effect of our implementation.

Spatial Data is the cost of maintaining and copying spatial object attributes. For the optimized executions, this overhead includes the cost of maintaining the pointer dirty bits and previous index values. Spatial Checks is the cost of performing spatial checks. Temporal Data is the cost of maintaining and copying temporal object attributes. For the optimized executions, this overhead includes the cost of maintaining the additional counter variable. Temporal Checks is the cost of performing temporal checks.

For BC, Min-Span, and Partition, run-time resolved optimization paid off with a slightly lower execution cost for spatial checking. For Anagram, Backprop, and YACR-2, adding run-time checks resulted in a higher cost for spatial access checking; and in the case of Backprop, a higher overall execution overhead.

These programs demonstrate the trade-offs involved in providing run-time resolved optimization. Run-time optimization adds the extra overhead of copying, maintaining, and checking the extra safe pointer state. If this added overhead, plus the overhead of the required checks, is greater than doing all the checks, there is no advantage to run-time check optimization. With faster checks, compile-time optimization, and spatially complex programs, this trade-off becomes even more acute. Since Anagram, Backprop, and YACR-2 must execute many of their checks $(39 \%, 67 \%$, and $86 \%$ respectively), they do not benefit from the run-time optimizations. For $Y A C R-2$, the effects are much less pronounced because dereferences are much less frequent (as shown in Table 1).

The second effect to observe when comparing the optimized to unoptimized execution costs is that the greatest benefit of run-time check optimization always comes from eliding temporal checks. In fact, adding run-time optimization for temporal checks caused a significant decrease in all execution overheads except Backprop. There are two facets to this result. First, temporal checks are very expensive (requiring an associative search), so eliding one has a great

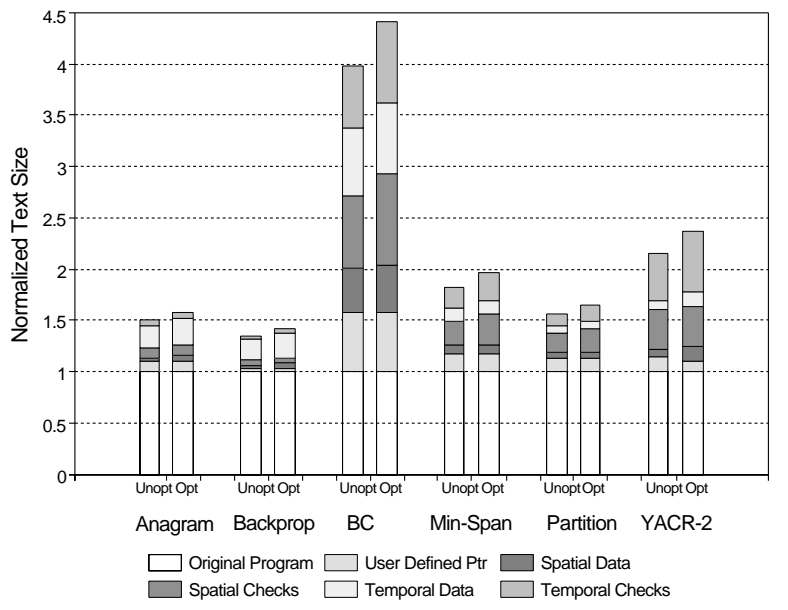

Figure 10: Text overheads.

performance advantage. Second, our run-time resolved optimization of temporal checks is very effective. Temporal checks are rarely required, even for $B C$ and $M i n-S p a n$, both of which free storage often. In the case of Backprop, adding run-time optimization for temporal checks resulted in an increased execution overhead. Backprop has only one dynamic object, an array, so temporal checking is relatively cheap without any optimization (the capability is always at the head of the hash bucket chain). In this case, the cost of maintaining the extra storage required for the free counter outweighs the cost of executing all temporal checks.

Adding checking code reduces the effectiveness of many traditional compiler optimizations. We inline all check code except for calls to ValidCapability() and abort(). These functions are both externally defined, so the compiler must make conservative assumptions as to what actions they take. This conservative approximation has the effect of limiting the effectiveness of many optimizations such as invariant code motion, register allocation, copy propagation, and common subexpression elimination. Neither of these functions produce any side-effects for normal executions. Hence, better compiler integration, i.e., providing a special channel of communication between the safe program generator and the compiler optimizer, would certainly increase the performance of our safe executions. ${ }^{4}$

Text size overheads are shown in Figure 10. All checking code, except the capability routines and what the $\mathrm{C}++$ compiler extracts for expression simplification, is inlined into the original program text. Surprisingly, the text overheads are quite small; $35 \%$ to $300 \%$ for the unoptimized executables and $41 \%$ to $340 \%$ for the run-time optimized programs. The text sizes for the run-time optimized programs are larger due to additional code required for maintaining, copying, and checking the extra object attributes. As shown by comparing Table 1 and Figure 10, there is a strong correlation between static dereference density and the resulting text overhead.

The data size overheads, shown in Figure 11, are measured as the total size of initialized (.data) and uninitialized (.bss) data segments plus the size of the heap segment when the program terminates execution. The data size overheads on the stack were not measured. All programs, except Min-

\footnotetext{
${ }^{4}$ Many compilers, e.g. GNU $g c c$, already understand the special semantics of abort () and use this inter-procedural information to improve optimizations. We should be able to achieve the same results for ValidCapability().
} 


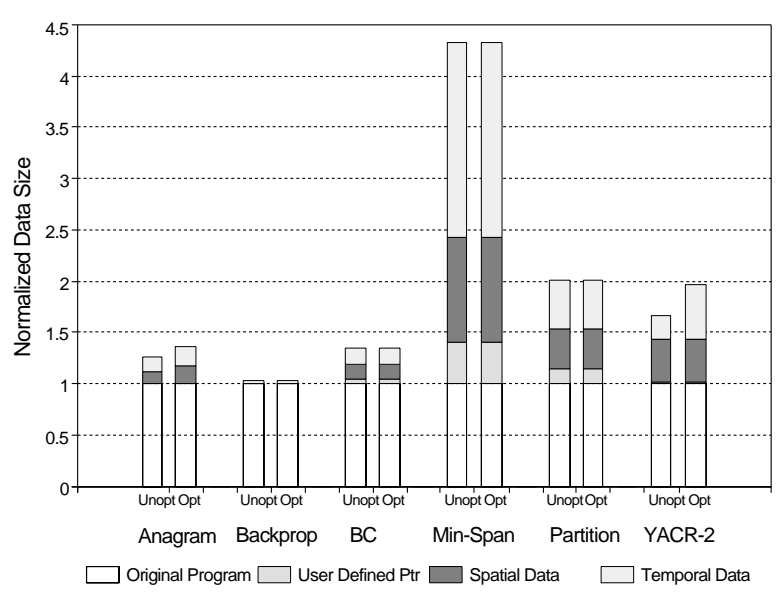

Figure 11: Data overheads.

Span, have data size overhead below 100\%. Backprop has the lowest overhead (less than $5 \%$ ) because most of its storage is large global arrays which do not require any object attributes. Min-Span has the highest overhead (330\%), which stems from the high density of pointers in its heap allocations, most of which contain eight pointers and three integers. Some of the run-time optimized programs have slightly larger overheads due to the additional object attributes.

To summarize the main points of our results:

- Execution overheads, even without compile-time optimization, are low enough to make our methodology useful during program development. However, the overheads are not likely low enough that programmers would release software with checking enabled. We are currently exploring the use of compile-time optimization and better compiler integration as means of increasing the performance of our approach.

- The largest contributing factors to execution overhead are 1) safe pointer structures are not register allocated, and 2) many traditional optimizations fail with the addition of checks. Other performance losses are attributed to the $\mathrm{C}++$ compiler simplifying expressions through the use of static functions, and, due to a bug in the $\mathrm{C}++$ compiler, the need to include the type size of the referent in the object attributes. None of these difficulties are without recourse, however. Better integration between the safe compiler and the optimizer could fix most problems.

- Dynamically eliding spatial checks is generally ineffective, primarily because maintaining the extra state, and checking it, quickly outweighs the cost of executing all checks. Our spatial check is very cheap to execute, and pointer intensive programs tend to execute most of the checks anyway.

- Temporal checks, on the other hand, are very expensive to perform and are rarely required, so run-time optimization shows to be beneficial in most cases.

- The text and data size overhead are generally quite low. The text overheads for all programs with run-time optimization, range from $41 \%$ to $340 \%$, with all but two below $100 \%$. Data overheads range from $5 \%$ to $330 \%$, with all but one below $100 \%$. Run-time optimized executions have slightly larger text and data sizes.

\section{Related Work}

Our first attempt at creating a safe programming environment for $\mathrm{C}$ employed reference chaining. The technique is similar to that used by many "smart pointer" implementations [EP91, Gin92]. The idea is to insert any pointer which is generated either through use of explicit storage allocation, e.g., malloc(), the reference operator (' $\&$ '), or assignment into a reference chain rooted at the referent. When a pointer value is destroyed, e.g., through assignment, storage deallocation, or at procedure returns, the pointer is removed from the reference chain. This technique has a number of useful properties. First, it is possible to ensure temporal safety by destroying all pointer values when a referent is freed - just march down the reference chain assigning NULL to all pointers. Second, if a destructed pointer value is the last value in the referent's reference chain, a storage leak has occurred and it is detected immediately. Unfortunately, this technique cannot be made to work reliably in C. It is relatively easy for the programmer to subvert the checking mechanism through recasting and typeless calls to free(). Storage leak detection also fails in the presence of circular references. The safe programming technique described in this paper is significantly more reliable because its correctness does not rely on tracking pointer values.

Some researchers have recently proposed providing complete program safety through limiting the constructs allowed in the language. The main thrust of this work is the design of languages that support garbage collection reliably and portably. In [ED93], a safe subset of $\mathrm{C}++$ is defined. The safe subset does not permit any invalid pointers to be created; this restriction, for example, precludes the use of any explicit pointer arithmetic. The safe subset also requires some checking, but much less than our checking technique requires. Languages which can easily be made totally safe have existed for a long time; for example, many FORTRAN implementations provide complete safety through range checking. However, these languages tend to be less expressive than intrinsically unsafe languages such as $\mathrm{C}$ or $\mathrm{C}++$. We felt that it was important not to restrict the expressiveness available to the programmer. Our checking technique is not limited by the language upon which it is applied, it can be applied successfully to compiled or interpreted languages with subscripted and mutable pointers, local references, and explicit and type-less dynamic storage management.

Table 2 details our work (Safe-C) and five other published systems that support memory access checking.

Hastings' and Joyce's Purify [HJ92] is a commercially available memory access checking tool. It is particularly convenient to use because it does not require program source - all transformations are applied to the object code. $P u$ rify supports both spatial and temporal access error checking to heap storage only through the use of a memory state map which is consulted at each load and store the program executes. Purify also provides uninitialized read detection, and storage leak detection through a conservative collector [Boe93, BW88]. Spatial access errors are detected by bracketing both ends of any heap allocation with a "red zone". These zones are marked in the memory state map as inaccessible. When a load or store touches a red zone a memory access error is flagged. Temporal access errors are detected by setting the memory state of freed storage to inaccessible. Purify cannot detect all memory access errors. For example, accessing past the end of an array into the region of the next variable, or accessing freed storage that has been reallocated cannot be detected. These limitations occur because Purify does not determine the intended referent of memory 


\begin{tabular}{|c|c|c|c|c|c|}
\hline \multirow[t]{2}{*}{ Name } & \multirow[t]{2}{*}{ Environment } & \multirow[t]{2}{*}{ Method } & \multicolumn{3}{|c|}{ Error Model } \\
\hline & & & Spatial Checks? & Temporal Checks? & Extensions \\
\hline Safe-C & $\mathrm{C} / \mathrm{C}++$ & $\begin{array}{l}\text { source-to-source } \\
\text { translation }\end{array}$ & yes* & yes* & errant free's \\
\hline $\begin{array}{l}\text { Purify } \\
\text { [HJ92] }\end{array}$ & object files & $\begin{array}{l}\text { object code } \\
\text { translation }\end{array}$ & $\begin{array}{l}\text { yes } \\
\text { limited to heap }\end{array}$ & $\begin{array}{l}\text { yes } \\
\text { limited to heap }\end{array}$ & $\begin{array}{l}\text { errant free's, uninitialized } \\
\text { reads, storage leaks }\end{array}$ \\
\hline $\begin{array}{l}\text { RTCC } \\
{[\text { Ste92] }}\end{array}$ & $\mathrm{C}$ & $\begin{array}{l}\text { safe } \\
\text { compiler }\end{array}$ & yes* & no & \\
\hline $\begin{array}{l}\text { CodeCenter } \\
\text { [KLP88] }\end{array}$ & $\mathrm{C} / \mathrm{C}++$ & interpreter & yes* & yes & $\begin{array}{l}\text { errant free's, uninitialized reads, } \\
\text { dynamic type checking, etc. }\end{array}$ \\
\hline $\begin{array}{l}\text { Bcc } \\
{[\text { Ken83] }}\end{array}$ & $\mathrm{C}$ & $\begin{array}{l}\text { source-to-source } \\
\text { translation }\end{array}$ & yes* & no & $\begin{array}{l}\text { alignment checks, } \\
\text { overflow checks }\end{array}$ \\
\hline $\begin{array}{l}\text { UW-Pascal } \\
\text { [FL80] }\end{array}$ & Pascal & $\begin{array}{l}\text { safe } \\
\text { compiler }\end{array}$ & yes* & yes & $\begin{array}{l}\text { errant free's, } \\
\text { arithmetic faults, etc. }\end{array}$ \\
\hline
\end{tabular}

Table 2: Comparison of memory access checking work. Entries with an asterisk $(*)$ indicate that the method detects all errors for that particular error class.

accesses - it can only verify that the accessed storage is active. Our checking technique, on the other hand, can detect all memory access errors because it tracks not only the state of storage, but also the intended referents of all pointer values. To increase the effectiveness of temporal error checking, Purify "ages" the heap, holding freed storage in the heap free list longer than needed. This aging increases the storage requirements of programs that use the heap. The primary disadvantage of our technique compared to Purify is that we require source code before any checking can be implemented; thus, source code is required if libraries are to be checked. Our technique is also not portable across languages, that is, a given implementation must be tailored for a specific language. However, our technique is quite portable across different platforms, especially if implemented as a source-to-source translator. Although Purify is portable across languages (on a given platform), it is not portable across platforms.

Steffen's RTCC [Ste92] extended the functionality of the $\mathrm{C}$ language compiler PCC to include spatial error checking. $R T C C$ attaches object attributes to pointers in a fashion similar to our technique; it does not, however, detect temporal access errors, nor does it explore the use of check optimization. Our checking technique finds both spatial and temporal access errors, and incorporates run-time and compile-time optimizations through which access checks can be elided. In the implementation of $R T C C$ the issue of interfacing to library and system calls is addressed through encapsulation; Steffen also augmented $s d b$ to provide users with transparent debugging support.

CodeCenter [KLP88] is an interpreted C language environment. The error checking provided is very rich - it detects many memory access errors as well as provides dynamic type checking (i.e., the type of the last store to memory must match the type of subsequent loads), uninitialized read detection, errant free detection, and other useful checks. The published information describing CodeCenter is somewhat ambiguous as to how it implements memory access checking. Object attributes (namely, type and size) are attached to all storage when it is initialized. If a reference is made to storage, it appears that the base and size attributes, associated with the referent storage, are also attached to the pointer value. Using this information, CodeCenter provides complete coverage for spatial access errors. However, it does not employ a capability based temporal checking scheme, so it is (sometimes) possible to access freed storage after it has been reallocated for another purpose. Temporal access checking can also fail for pointer references to local variables. Because our checking technique employs a capability based scheme, it never misses temporal access errors. The primary disadvantage of CodeCenter is its resource requirements. Since programs run in an interpreter, the execution overheads may discourage its use, and in the case of long running programs, may preclude its use. Due to our use of compile-time instrumentation, resource requirements are significantly lower. Compile-time instrumentation also allows us to employ static check optimizations.

Kendall's Bcc [Ken83] is a commercial source-to-source translator for the $\mathrm{C}$ language. It supports spatial error checking, but temporal error checking is limited to NULL checks at all pointer dereferences. The published information on $B c c$ does not specify how the checking is implemented, however, one figure in the paper, showing the output of the translator, suggests that base and bound object attributes are attached to all pointer values.

Fischer and LeBlanc's UW-Pascal compiler [FL80] supports both temporal and spatial error checking. However, the lack of mutable pointers and dynamically sized arrays makes access checking much easier. While $U W$-Pascal detects all spatial access errors, temporal access errors may not be detected if storage is reallocated. Use of our checking technique is not limited by the expressiveness of the language; that is, it can be applied successfully to compiled or interpreted languages with subscripted and mutable pointers, local references, unions, and explicit and typeless dynamic storage management.

A closely related area of work, which can benefit from our safe programming technique, is storage leak detection [Boe93, BW88, ZH88]. A storage leak is any storage to which the program can no longer generate a name. These leaks occur when the last accessible pointer to a heap object is overwritten. Without the ability to generate a name to the heap object, it cannot be freed, hence it has "leaked" out of the heap.

For languages like $\mathrm{C}$ and $\mathrm{C}++$, leak detection is commonly implemented with a conservative collector. A conservative collector sweeps memory looking for unreferenced storage. Because it is difficult to know where all pointers are located, the collector makes the conservative assumption that all program accessible (non-heap) storage contains pointers. It then uses a traditional mark and sweep collection method. While 
effective, this method has some drawbacks. First, storage leak detection is not immediate, it is usually applied only when the programmer demands it or when the program completes execution. Thus, for it to be useful, some dynamic information, like a partial call chain, must be kept with allocations, in order for the programmer to deduce the circumstances under which the storage leak occurred. Second, the conservative pointer assumption can cause non-pointer values to be mistaken as pointer values which seem to reference heap storage. These false hits can hide a storage leak. The problem is aggravated by large storage allocations because it is more likely that non-pointer values inadvertently reference them; unfortunately, it is these large allocation leaks that we would most like to find. Third, if the program hides pointers, for example, by encoding type information in the upper bits of a pointer, or does not keep all pointers within the bounds of memory allocations, the collector may regard heap storage as a leak when it is still in use.

These false leaks cannot occur under our checking scheme because the base field always holds a pointer to the head of the allocation, and the program cannot manipulate this value. We can also address the problem of false hits, that is, non-pointer values which appear to reference heap storage, by applying safe pointer invariants to possible references. One trivial test is to ensure that both the capability and the free counter values of the possible reference are valid. If an incrementing counter is used for each, each value should be less than the current counter value. To summarize, using a conservative collector to detect storage leaks with our safe programming technique makes the process intrinsically more reliable by eliminating false leaks and reducing the possibility of false hits.

\section{Conclusions}

In this paper, we presented a pointer and array access checking technique that provides complete error coverage through a simple set of program transformations. Our technique, based on an extended safe pointer representation, has a number of novel aspects. It is the first technique that detects all spatial and temporal access errors. Its use is not limited by the expressiveness of the language; that is, it can be applied successfully to compiled or interpreted languages with subscripted and mutable pointers, local references, unions, and explicit and type-less dynamic storage management. We showed the transformations required in the context of the $\mathrm{C}$ language, and also developed run-time and compile-time check optimization frameworks. Finally, we described our prototype implementation, and used it to analyze the execution, text and data size overheads of six non-trivial, pointer intensive $\mathrm{C}$ programs. We showed that performance with only run-time resolved optimizations was quite good. For all six programs, instruction execution overheads ranged from $130 \%$ to $540 \%$, with text and data size overheads typically below $100 \%$. The primary factors to performance degradation in safe programs are the lack of safe pointer register allocation and ineffective optimization in the presence of check functions. We see the solution to these problems as better integration between the safe compiler and the code generator.

Our prototype implementation, while successful at showing the viability of our compile-time safe programming methods, leaves many questions of efficiency and usability unanswered. We are addressing these issues with the development of our fully automatic, optimizing Safe- $C$ compiler.

\section{Acknowledgements}

We thank Jim Larus, Tom Ball, Alain Kägi, and Alvy Lebeck for numerous discussions which helped shape this paper. Also, thanks to Mary Baker, Hans-Juergen Boehm, John Ellis, Mark Sullivan, Mark Weiser, Ben Zorn, and the anonymous referees for providing useful comments and directing us to relevant references.

\section{References}

[ASU86] A.V. Aho, R. Sethi, and J.D. Ullman. Compilers: Principles, Techniques, and Tools. Addison-Wesley, Reading, MA, 1986.

[Boe93] Hans-Juergen Boehm. Space efficient conservative garbage collection. Proceedings of the ACM SIGPLAN'93 Conference on Programming Language Design and Implementation, 28(6):197-204, June 1993.

[BW88] Hans-Juergen Boehm and Mark Weiser. Garbage collection in an uncooperative environment. Software - Practice and Experience, 18(9):807-820, September 1988.

[ED93] John R. Ellis and David L. Detlefs. Safe, efficient garbage collection for C++. Technical Report 102, DEC Systems Research Center, June 1993.

[EP91] D. R. Edelson and I. Pohl. Smart pointers: They're smart but they're not pointers. Proceedings of the 1991 Usenix C ++ Conference, April 1991.

[FH88] Anthony J. Field and Peter G. Harrison. Functional Programming. Addison-Wesley Publishing Company, 1988.

[FL80] Charles N. Fischer and Richard J. LeBlanc. The implementation of run-time diagnostics in Pascal. IEEE Transactions on Software Engineering, SE-6(4):313-319, 1980.

[Gin92] Andrew Ginter. Design alternatives for a cooperative garbage collector for the $\mathrm{C}++$ programming language. Technical Report 91/417/01, Department of Computer Science, University of Calgary, 1992.

[Gup90] Rajiv Gupta. A fresh look at optimizing array bound checking. Proceedings of the ACM SIGPLAN'90 Conference on Programming Language Design and Implementation, pages 272-282, June 1990.

[HJ92] Reed Hastings and Bob Joyce. Purify: fast detection of memory leaks and access errors. Proceedings of the Winter Usenix Conference, 1992.

[Ken83] Samuel C. Kendall. Bcc: Runtime checking for C programs. Proceedings of the Summer Usenix Conference, 1983.

[KLP88] Stephen Kaufer, Russel Lopez, and Sesha Pratap. Saber$\mathrm{C}$ : an interpreter-based programming environment for the $\mathrm{C}$ language. Proceedings of the Summer Usenix Conference, pages $161-171,1988$.

[Lar93] James R. Larus. Efficient program tracing. IEEE Computer, 26(5):52-61, May 1993.

[Lee91] Peter Lee, editor. Topics in Advanced Language Implementation. The MIT Press, Cambridge, MA, 1991.

[MFS90] Barton P. Miller, Lars Fredriksen, and Bryan So. An empirical study of the reliability of Unix utilities. Communications of the ACM, 33(12):32-44, December 1990.

[Ros87] Graham Ross. Integral C - a practical environment for $\mathrm{C}$ programming. In Proceedings of the $A C M S I G$ SOFT/SIGPLAN Software Engineering Symposium on Practical Software Development Environments (SIGPLAN Notices), pages 42-48. Association for Computing Machinery, January 1987.

[SC91] Mark Sullivan and Ram Chillarege. Software defects and their impact on system availability - a study of field failures in operating systems. Digest of the 21st International Symposium on Fault Tolerant Computing, pages 2-9, June 1991.

[Ste92] Joseph L. Steffen. Adding run-time checking to the Portable C Compiler. Software - Practice and Experience, 22(4):305-316, 1992.

[ZH88] Benjamin Zorn and Paul Hilfinger. A memory allocation profiler for $\mathrm{C}$ and Lisp programs. Proceedings of the Summer Usenix Conference, pages 223-237, 1988. 